\title{
山莨蓉碱对磷脂脂质体膜 $\mathrm{Ca}^{2+}$ 通透性的影响
}

\author{
谢静平黄芬 \\ （中国科学院生物物理研究所，北京）
}

山莨菅碱是莨莒类植物中提取分离的新药, 过去结果表明, 药物能降低磷脂膜的有序 度: [1], 诱发磷脂酰乙醇胺及一些带电荷磷脂形成非脂双层的 $\mathrm{H}_{11}$ 结构 ${ }^{[2]}$. 此外, 还对大鼠突螌 膜上多种 ATP 酶产生抑制作用 ${ }^{[3]}$. 生理实验业已表明, 山莨若碱作用于神经系统主要抑制 神经递质乙酰胆碱的释放及与其受体的结合, 而乙酰胆碱的释放主要是由神经元内 $\mathrm{Ca}^{2+}$ 来 调节的, 为此, 本文以注射法制备的单层脂质体为模型, 研究山莨宕碱对中性磷脂二棕悯酰磷 脂酰胆碱 (DPPC) 及中性磷脂混以不同克分子比酸性碱脂二棕栒酰磷脂酸 (DPPA) 脂质体 膜 $\mathrm{Ca}^{2+}$ 通透性的影响.

\section{一、材料与方法}

DPPC，DPPA， $A_{23187}$ (钙盐) 及偶氮钤 III 均为美国 Sigma 公司产品, 㳦聚糖凝胶 G50 , 瑞典 Pharmacia 公司产, 山茛菅碱(氢澳酸盐), 成都第一制药厂出品. 其它试剂均为分析 纯.

1. 大单层脂质体 (LUV) 的制备基本参照 Deamer 和 Bangham ${ }^{[4]}$ 的方法,略有修 改.称取约 $5 \mathrm{mg} \mathrm{DPPC}$ 于小烧杯内, 加入所需克分子比的酸性磷脂 DPPA, 然后按 $1.5 \mathrm{mg} / \mathrm{ml}$ 磷脂加人乙醚: 甲醇 $(V / V)=9: 1)$ 将磷脂分散. 用一注射器吸出, 以 $0.25 \mathrm{ml} / \mathrm{min}$ 的速度: 注射到恒温在 $70^{\circ} \mathrm{C}$ 的 Tris 缓冲液 $(25 \mathrm{mmol} / 1 \mathrm{Tris}-\mathrm{HCl}, 50 \mathrm{mmol} / 1 \mathrm{NaCl}, 50 \mathrm{mmol} / 1 \mathrm{KCl}$,

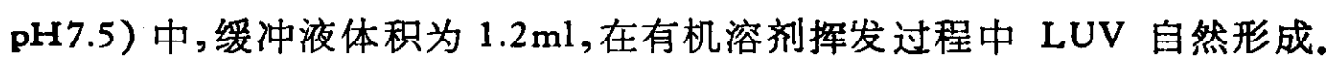

\section{2. 脂质体膜 $\mathrm{Ca}^{2+}$ 通透性的测定 参昭 Smaal 等 ${ }^{[5]}$ 基于 Weissman 等 ${ }^{[6]}$ 所修改的方} 法, 以偶氮钤 III(AIII) 作为 $\mathrm{Ca}^{2+}$ 探针，注射法制备脂质体过程中， Tris 缓冲液中加入 2.5 . $\mathrm{mmol} / 1$ 的 AIIl, 脂质体在室温下超声清洗器中超声 $5 \mathrm{~min}$ 后, 用台式离心机 $10000 \mathrm{r}$ 离心 $20^{\prime}$ $\mathrm{min}$, 去掉上清, 收集脂质体沉淀 (磷脂浓度为 $0.1 \mathrm{mg} / \mathrm{ml}$, 加入所需量的山莨荢碱, 在脂质体相: 变温度以上保温 $1.5 \mathrm{~h}$, 使药物炊入膜内, 然后柱层析 (Sephadex G-50,18 $181 \mathrm{~cm}$ ), 除去末 圈套的脂质体外探针, 游离的药物及溶液中的微量有机溶剂, 在洗脱峰处收集 $1.4 \mathrm{ml}$ (磷脂㳊 度约 $2 \mathrm{mg} / \mathrm{ml}$, 收集后的样品立即加入 $2.5 \mathrm{mmol} / 1$ 的庶糖以维持脂质体内、外的渗透压. 测昷 $\mathrm{Ca}^{2+}$ 通透性时在体系中加人 $2.0 \mathrm{mmol} / 1$ 的 $\mathrm{Ca}^{2+}$, 记录 AIII 吸收峰的变化便可反映出 $\mathrm{Ca}^{2+}$ 自 向穴穿膜运动的速度和性质, 光谱测量在 Hitachi 557 双波长双光束分光光度计上进行: 测量时温度控制在 $30^{\circ} \mathrm{C}$.

本文 1987 年10月13 日收到. 
政脂含量的测定采用定无机磷的方法, 详细参见文献 [7].

\section{二、结果与讨论}

偶瓷钤 III 是一种水溶性无毒的金属生色剂, 它在结合 $\mathrm{Ca}^{2+}$ 后颜色从红到蓝发生显著 变化, 且两者差光谱的克分子消光系数很高 (达 $10^{4}$ ), 与其它金属离子比较, 它对钻有选择专一 性 ${ }^{[6]}$. 在低 $\mathrm{Ca}^{2+}$ 和 AIII 浓度时 $(<100 \mu \mathrm{mol} / 1)$ 两者形成 $1: 1$ 的复合物 ${ }^{[0]}$. 对一定量的 指示剂浓度, 差光谱的光吸收值随着 $\left[\mathrm{Ca}^{2+}\right]$ 的增加而线性增加, 差光谱在 $\lambda_{650}$ 处（相对于 $\lambda_{700}$ ) 的光吸收值正比于与 $\mathrm{Ca}^{2+}$ 结合的指示剂 $\mathrm{A} I 1 \mathrm{I}$ 量. 测定脂质体膜 $\mathrm{Ca}^{2+}$ 通透性时, 指示 判 AIII 圈套在脂质体内, 在人为建立 $\mathrm{Ca}^{2+}$ 梯度差后, 实验结果表明脂双层对 AIII 不通透, 仅 $\mathrm{Ca}^{2+}$ 穿膜转运到脂质体内与指示剂结合后才引起颜色变化. 因此, 监示差光谱双波长 $\lambda_{700-650}$ 光吸收值随时间的变化可反映出 $\mathrm{Ca}^{2+}$ 穿膜运动的性质和速率.

以中性磷脂 DPPC 及 DPPC 混以不同克分子比的酸性磷脂 DPPA 按注射法形成的脂 质体对 $\mathrm{C}_{2}{ }^{2+}$ 的通透性见图 1, 从比较基本稳定后的纵轴截距和到此所需的时问看出, 中性

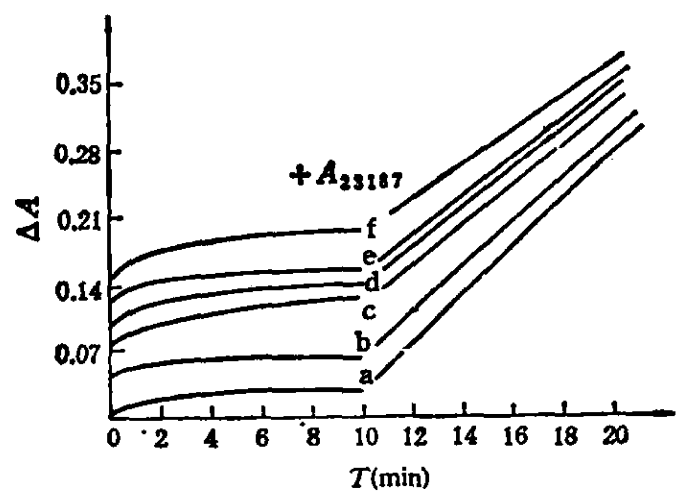

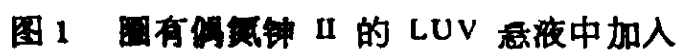
$2 \mathrm{mmol} / 1 \mathrm{Co}^{2+}$ 后 $\mathrm{C}_{2}{ }^{3+}$-AIII 复合物的形成

等质体量洨中含 $50 \mathrm{menol} / 1 \mathrm{KCl}, 50 \mathrm{mmol} / 1 \mathrm{NaCl}$ 㕲 $25 \mathrm{mmol} / 1 \mathrm{Tris}-\mathrm{HICl}(\mathrm{pH} \mathrm{7.5)}$. 碚脂浓度约 2 $m g / \mathrm{ml}$. 脂感洨在波长 $650 \mathrm{~nm}$ 处(似 $700 \mathrm{~nm}$ 为考) 的吸收变化被连续扫描记录. $a, b, c, d, e$ 和 $f$ 分 别对应于 DPPA/DPPC (克分子比)值 $0,5 \%, 10 \%$, $15 \%, 20 \%$ 和 $25 \%$. 湿度 $30^{\circ} \mathrm{C}$

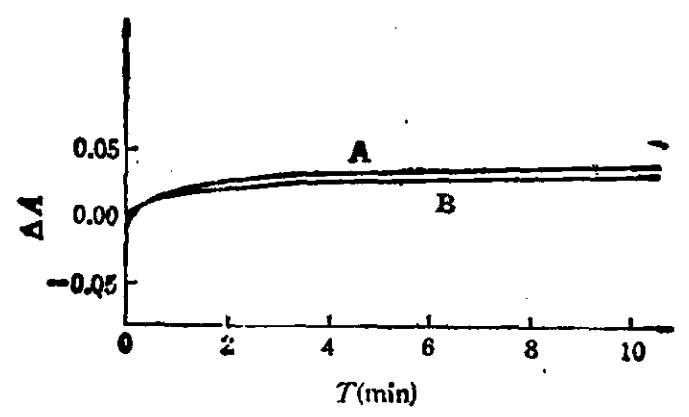

因 2 将偶资钤 III 圈套在脂质 体内作监示

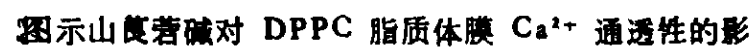

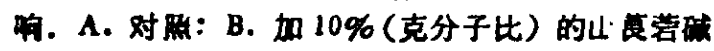

磷脂 DPPC 形成的脂质体对 $\mathrm{Ca}^{2+}$ 几乎不通 透, 而混有酸性磷脂 DPPA 的脂质体具有 $\mathrm{Ca}^{2+}$ 通道功能。且随着酸性磷脂含量的增加其通透 性增加. 酸性磷脂对 $\mathrm{Ca}^{2+}$ 通透性是孔道式的, 这与 $\mathrm{Ca}^{2+}$ 载体 (如 $\mathrm{A}_{23 \mathrm{s8}}$ ) 相区别. 离子载体 借助于离子梯度, 将 $\mathrm{Ca}^{2+}$ 从膜的一侧传送到 另一侧, 有一相当的时间过程. 而酸性磷脂形 成的 $\mathrm{Ca}^{2+}$ 通道,在建立 $\mathrm{Ca}^{2+}$ 梯度后, $\mathrm{Ca}^{2+}$ 急 剧拥人, 但很块 (小于 $30 \mathrm{~s}$ ) 缓慢而趋于平稳. 平稳后加入离子载体 $\mathrm{A}_{23160}$ 可使差光谱 $\mathrm{OD}$ 值 进一步升高. 说明脂质体内 $\mathrm{Ca}^{3+}$ 并未达到饱 和. 由此反映出酸性磷脂所诱发 $\mathrm{Ca}^{2+}$ 通道的 开关性. 即对一定量酸性磷脂形成的 $\mathrm{Ca}^{2+}$ 通 道,在一定量的 $\mathrm{Ca}^{2+}$ 进人后,可能将自行封闭 其通道.

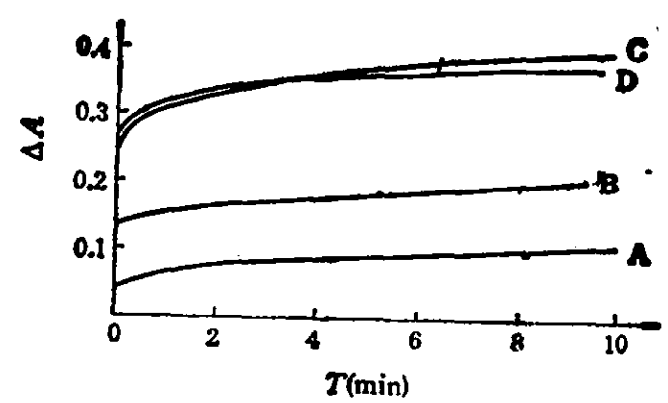

国 3 山茛宕碱通过与酸性磷脂作用对 DPPC-DPPA 脂质体膜 $\mathrm{Ca}^{2+}$ 通适生的影絮

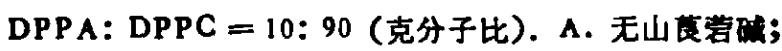

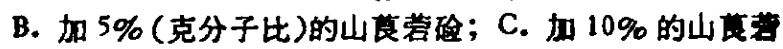
碳；D. 加 $20 \%$ 的山茛营破. 进一步细节见正文 


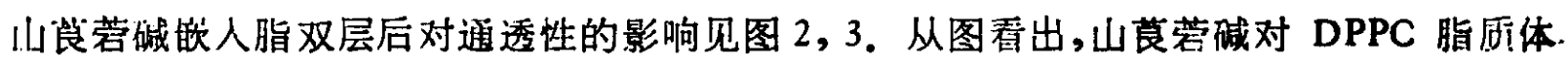
$\mathrm{Ca}^{3+}$ 的通透性无明显影响。但对混有酸性磷 脂的脂质体膜, 药物能促进和加强其通透功能. 在药物浓度低于酸性磷脂所占克分子比时，随 着约物浓度的增加，通透性增加．当药物比例 等于或通过酸性磷脂比例时, 药物的增加不再 使通透性进一步加强. 说明药物在脂双层中先 选择性:地和酸性磷脂作用. 这点也可从药物分 子结构和脂双层构象上得到认识. 综合以前的 结果, 我们推论出: 山莨宕碱与磷脂的相互作 用可能如图 4 所示, 药物可解离的三级胺与磷 脂分子的磷酸基团相平行, 相互静电吸引。中 间部位的羟基和磷脂甘油骨架 $\boldsymbol{\alpha}$ 碳链上的㷇基 相互作用, 这点同胆固醇与磷脂分子的相互作 用 ${ }^{[212]}$ 类似，而苯环深人到脂酰链流水区. 对酸 性磷脂 DPPA，它极性头部带两个解离基团且 空间位阻很小,容易与药物 $1: 1$ 结合而形成分 相域. 当药物浓度高于酸性磷脂 DPPA 所占 比例时, 约物才作用于 DPPC 脂双层, 从而不

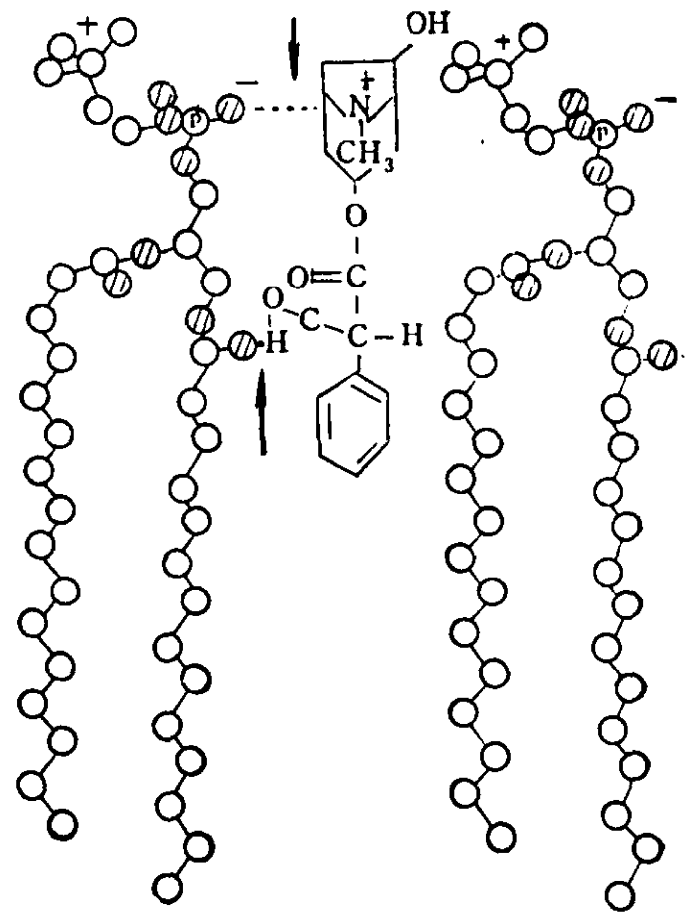

图 4 山莨营碱与磷脂分子的可能作用方式 图中磷脂分子为 PC

对通透性产生进一步影响. 磷脂酸 ${ }^{[9]}$ 和被氧化的脂肪酸 ${ }^{(00)}$ 具有 $\mathrm{Ca}^{2+}$ 通道功能已有报道, 但机 理尚不清楚. 有人提出, 非脂双层六角形 II 结构能充当 $\mathrm{Ca}^{2+}$ 通道 ${ }^{[11]}$. 山莨茓碱能促进酸性 磷脂的 $\mathrm{Ca}^{2+}$ 通道功能, 或许与它能诱发酸性磷脂形成非脂双层结构有关. 它可能产生的生 理影响有待于结合各种离子泵作系统研究后才能作出定论, 目前, 进一步研究正在进行中.

\section{参考文献}

(1] Hwang, F. et al., Biochin. Biophys, Acra, 736(1983), 220-225.

[2] 何埼、黄茒, 生物化学术先, 2(1986), 63-66.

[3] Hwang, F. et al., Biochim. Biophys. Acta, 860(1986), 713-716.

[4] Deamer, D. \& Bangham, A. D., Biochim. Biophys. Acta, 443(1976), 629-634.

[5] Smaal, E. B. el al., Biochim. Biophys. Acra, 816(1985), 418-422.

[6] Weissmann, G. et al., Proc. Natl. Acad. Sci. USA, 73(1976), 510-514.

[ 7$]$ Clien, T. P. S. et al., Anal. Chem., 28(1956), 1756-1758.

I 81 Bauee, P. J., Anal. Biochem., 110(1981), 61-72.

I01 Nayer, R. et al., Biochim. Biophys. Acta, 777(1984), 343-346.

[10] Serhan, C. et al., J. Biol. Chem., 256(1981), 2736-2741.

[11] Harris, R. A. et al., Science, 212(1981), 1290-1291.

[12] Brockerholf, H., Lipids, 9(1974), 645-650. 